\title{
Autologous fecal transplant for the treatment of microcolon due to diversion colitis
}

\author{
C. A. Donahue ${ }^{1}$ D $\cdot$ V. Chaudhry ${ }^{2} \cdot$ N. Mantilla ${ }^{2}$
}

Received: 7 September 2021 / Accepted: 18 October 2021 / Published online: 9 November 2021

(c) Springer Nature Switzerland AG 2021

\section{Dear Sir,}

We read with interest the correspondence by Grundling and colleagues [1] and would like to share with the readers a similarly successful case of treatment of diversion colitis complicated by microcolon with a prolonged autologous fecal transplant.

We present a case of a 20-year-old man who presented to our hospital to pursue colostomy reversal. The patient was a victim of a gunshot wound 3 years prior resulting in transient paraplegia and colonic injury. He underwent repair of two sigmoid colonic injuries and diverting colostomy. $\mathrm{He}$ returned to the operating room 8 months later for colostomy reversal which was complicated by prolonged ileus necessitating a return to the operating room for right upper-quadrant end colostomy and left upper-quadrant mucous fistula. Three years later, after immigrating to the United States, he was seen in our clinic to discuss reversal.

It is our practice to perform a gastrografin enema study and colonoscopy prior to colostomy and ileostomy reversals. His gastrografin enema revealed a diminutive sigmoid, descending and transverse colon leading up to the mucous fistula, measuring $21 \mathrm{~mm}$ in diameter (Fig. 1). There was a short segment of ascending colon which was opacified through the colostomy. Colonoscopy revealed disuse colitis without other abnormalities (Fig. 2).

Given the small caliber of the colon, we did not feel he would be able to undergo colostomy reversal without the need to perform a subtotal colectomy and ileorectal anastomosis. As a government-funded hospital serving under and uninsured patients, we do not have the resources to prescribe butyrate enemas. Therefore, we decided to treat him with

\footnotetext{
C. A. Donahue

Colleen.Donahue11@gmail.com

1 Medical University of South Carolina, 171 Ashley Avenue, Charleston, SC 29403, USA

2 John H Stroger Hospital of Cook County, Chicago, IL, USA
}

fecal transplant therapy to improve the caliber of the colon prior to reversal in an effort to salvage the distal colon. The patient was taught to inject fecal material into the mucous fistula 1-2 times a day. He was compliant with this therapy and was subsequently passing stool from his anus without incontinence. While the initial treatment plan was to continue enemas for 6 months followed by repeat gastrografin enema and colonoscopy, his repeat studies were delayed for an additional 6 months due to the coronavirus disease (COVID-19) pandemic. After a year of fecal therapy, the patient underwent repeat gastrografin enema and colonoscopy. Gastrografin enema revealed a residual atrophic colon with slight increase in caliber from 21 to $22 \mathrm{~mm}$ diameter (Fig. 1). Colonoscopy revealed healthy appearing mucosa with a small amount of stool in the rectum related to fecal enemas (Fig. 2).

The patient was brought to the operating room and underwent exploratory laparotomy with colostomy reversal and side to side, isoperistaltic ascending colon to transverse colon anastomosis. We performed a handsewn anastomosis given the caliber mismatch of the ascending and transverse colon. The mucosa and pliability of the transverse, distal and sigmoid colon appeared healthy and large enough to perform a colocolonic anastomosis which was preferred over an ileorectal anastomosis.

Postoperatively, the patient was maintained on a liquid diet for 2 weeks. He was having normal bowel movements without incontinence. He denied any significant bloating and did not have a prolonged ileus. He followed up in our clinic after 2 weeks and was advanced to a low fiber diet which he has been tolerating well.

Autologous fecal transplant is a safe, effective and affordable treatment for patients with diversion colitis. It has been shown to improve the endoscopic appearance of the colon as well as to act as a test of the functionality of the colon and rectum prior to performing ostomy reversal. Beside the above-mentioned case, Grudling similarly to Kalla et al. replicated the technique by performing fecal transplants over 
Fig. 1 Gastrografin enema demonstrating distal rectum and colon leading up to the mucous fistula. Image on the left represents the initial study prior to initiation of fecal transplant therapy. The image on the right shows a repeat gastrografin study after 1 year of fecal therapy
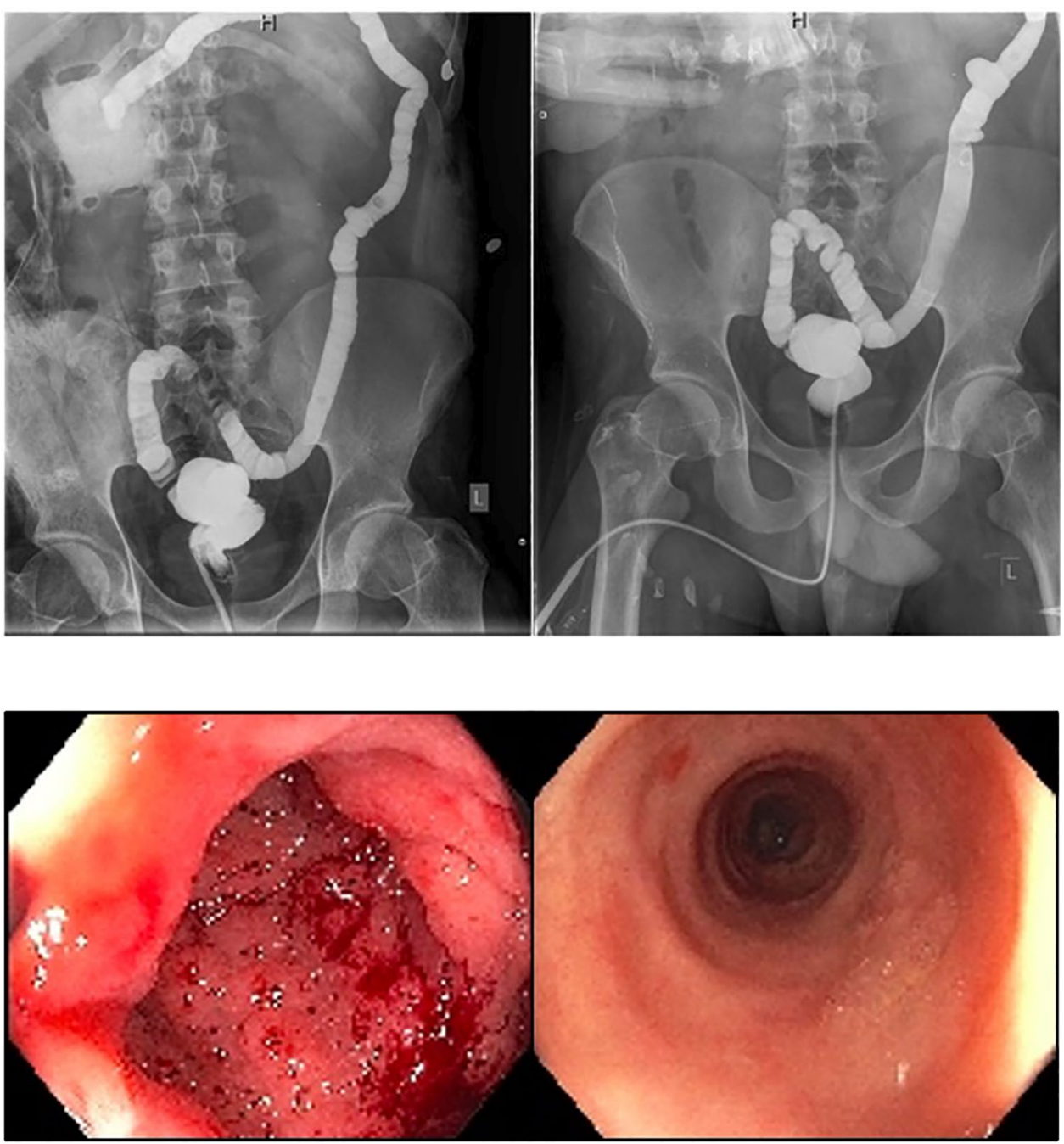

Fig. 2 Flexible sigmoidoscopy demonstrating caliber and appearance of the distal and sigmoid colon mucosa. The image on the left shows severe disuse colitis prior to initiating fecal therapy. The image on the right shows normal, healthy appearing mucosa after 1 year of fecal therapy the same time period, resulting in endoscopic and histological remission in a patient with diversion colitis refractory to other medical therapies [1,2]. Our patient demonstrated endoscopic and gross remission of diversion colitis after a year of treatment, although treatment was prolonged due to the COVID-19 pandemic.

For autologous fecal transplants to be successful in the treatment of diversion colitis, patients must be reliable and self-motivated to perform fecal transplants independently. Given the limited number of studies, further investigation is required to determine the optimal frequency and duration of treatment prior to performing reestablishment of intestinal continuity.

Author contributions All the authors made substantial contributions to the conception or design of the work; or the acquisition, analysis, or interpretation of data for the work; and drafting the work or revising it critically for important intellectual content; and final approval of the version to be published; and agreement to be accountable for all aspects of the work in ensuring that questions related to the accuracy or integrity of any part of the work are appropriately investigated and resolved.

Funding No funding was provided for this research or manuscript. This research did not receive any specific grant from funding agencies in the public, commercial, or not-for-profit sectors.

Availability of data and material Data sharing is not applicable to this article as no datasets were generated or analyzed during the current study.

Code availability Not applicable.

\section{Declarations}

Conflict of interest The authors declare no conflicts of interest, use of off-label or unapproved drugs or products, use of previously copyrighted material relevant to this manuscript.

Consent to participate Vernal informed consent was obtained from the participant included in the study. 
Consent for publication The patient has consented to the submission of the case report to the journal.

Ethical approval Ethics approval not required for the publication of case reports per the Cook County Health IRB.

\section{References}

1. Gundling F, Tiller M, Agha A, Schepp W, Iesalnieks I (2015) Successful autologous fecal transplantation for chronic diversion colitis. Tech Coloproctol 19:51-52. https://doi.org/10.1007/ s10151-014-1220-2

2. Kalla R, Pitt M, Sharma A (2019) The role of autologous fecal microbiota transplantation in diversion colitis: a case report. Inflamm Bowel Dis 25:E29-E30. https://doi.org/10.1093/ibd/ izy 270

Publisher's Note Springer Nature remains neutral with regard to jurisdictional claims in published maps and institutional affiliations. 